\title{
A Second Harmonic Self-Oscillating Mixer Incorporating Resonant Cell Structure
}

\author{
Leung Chiu ${ }^{1,2}$ and Quan Xue ${ }^{1}$ \\ ${ }^{1}$ Department of Electronic Engineering, City University of Hong Kong, Tat Chee Avenue, Kowloon, Hong Kong \\ ${ }^{2}$ Advanced Research and Development Center, Telefield International (Holdings) Limited, Unit 609-610, Bio-Informatics Centre, \\ Hong Kong Science Park, Hong Kong \\ Correspondence should be addressed to Leung Chiu, eechiuleung@yahoo.com.hk
}

Received 2 March 2012; Accepted 29 August 2012

Academic Editor: Giampiero Lovat

Copyright () 2012 L. Chiu and Q. Xue. This is an open access article distributed under the Creative Commons Attribution License, which permits unrestricted use, distribution, and reproduction in any medium, provided the original work is properly cited.

A downconverting second harmonic self-oscillating mixer (SOM) is developed for low-cost wireless communications applications. Incorporating resonant cell in the SOM, we can provide suitable oscillation for generating LO and terminations to all major unwanted mixing products, leading to high conversion gain design. The proposed SOM was measured with $8.5 \mathrm{~dB}$ downconversion gain at the RF frequency of $8.2 \mathrm{GHz}$ RF, LO frequency of $4.0 \mathrm{GHz}$, and IF frequency of $0.2 \mathrm{GHz}$. The proposed design achieves higher conversion gain than that of the SOM without resonant cell.

\section{Introduction}

Compact, inexpensive, and low-power-consumption frontend transceivers are the crucial elements in the future wireless systems. Self-oscillating mixer (SOM), which combines both functions of local oscillator (LO) and frequency conversion, will be a good candidate for the compact wireless systems. Over this decade, SOM has found interests in the many radio frequency $(\mathrm{RF}) /$ microwave transceivers, as the SOM exhibits smaller size and potentially lower overall DC power consumption compared to their conventional counterpart arrangement of separated active mixer and oscillator. Since the first field-effect transistor (FET) SOM was introduced in [1], a number of different variations of the SOM design have been demonstrated such as dual-gate and balanced FET SOM configurations [2, 3]. Moreover, in certain short-range applications, it is even possible to use a SOM with high conversion gain integrated into an antenna [4], thus allowing for the development of a lowcost and ultra-compact-size single-element RF/microwave transceiver.

Harmonic SOM is one of the extensions from the standard SOM technique: instead of mixing the RF signal with the fundamental LO frequency, one of its harmonics is used. A satisfactory isolation between the RF, LO, and IF can be easily achieved by simple filtering techniques. However, the performance such as conversion gain is not as good as the fundamental SOM. Recently, a third-harmonic SOM with high conversion gain has been developed pushing the stateof-the-art in modern SOM design towards high-order subharmonic architectures [5].

Recently, various works on resonant cells were found application of designing various microwave components [6]. In this paper, an improved resonant cell structure is proposed as a part of series feedback of SOM design. We can provide proper return paths for the RF, IF and other unwanted mixing products simultaneously, leading to a very high conversion gain design without sacrificing the circuit size.

\section{Proposed SOM Circuit}

Figure 1 shows the schematic diagram of the proposed second-harmonic SOM. The band-pass filter was designed with passband at the frequency of the RF $\left(f_{\mathrm{RF}}\right)$ and stop band at the frequency of the LO $\left(f_{\mathrm{LO}}\right)$. This band-pass filter functions as both the resonator of oscillation and the matching network for RF. The low-pass filter, which only allows IF to pass through, is connected at the collector 


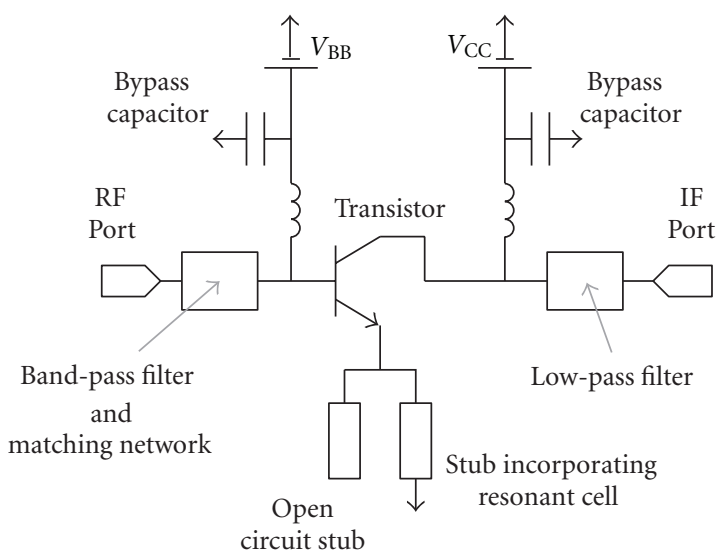

FIGURE 1: Schematic diagram of the proposed SOM.

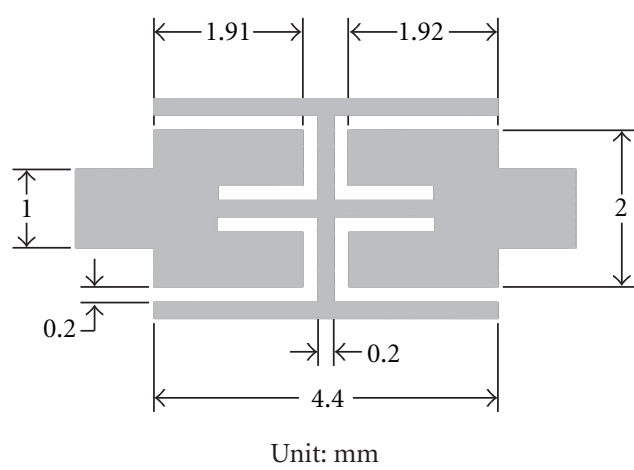

(a)

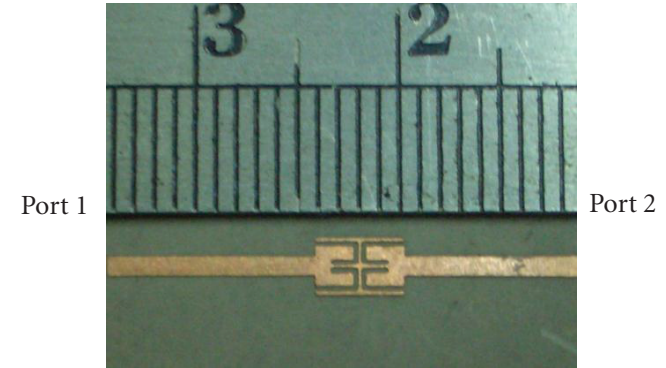

(b)

Figure 2: (a) Layout of the resonant cell with physical dimension. (b) Photograph of the fabricated resonant cell.

terminal of the transistor to suppress RF, LO, and spurious signals.

In order to have a proper return path for the signals and provide the series feedback for the oscillation, two stubs are connected to the two emitter terminals of the transistor. The modified resonant cell structure is incorporated into the stub. Figure 2 shows the layout and photograph of the resonant cell with physical dimensions. The resonant cell was fabricated on a piece of RT duroid 6002 dielectric substrate with a dielectric constant of 2.94 and substrate thickness of $0.381 \mathrm{~mm}$. All physical dimensions are tuned with the lower passband covering $f_{\mathrm{LO}}$ and the stop band covering $f_{\mathrm{RF}}$. Figure 3 shows the frequency response of the S-parameters of the resonant cell, where $4 \mathrm{GHz} \mathrm{LO}$ and $8.2 \mathrm{GHz}$ RF are chosen in this study.

The layout of the stub incorporating resonant cell is shown in Figure 4. With proper tuning of the electrical length of the two sections of the microstrip lines connected at both ends of the resonant cell, the input impedances of the stub incorporating resonant cell $Z_{\text {stub }}$ becomes capacitive at $f_{\mathrm{LO}}$ and $0 \Omega$ at $f_{\mathrm{RF}}$, respectively. Therefore, the stub incorporating resonance cell functions as a series feedback network at $f_{\mathrm{LO}}$ and short circuit at $f_{\mathrm{RF}}$, simultaneously. The open circuit stub

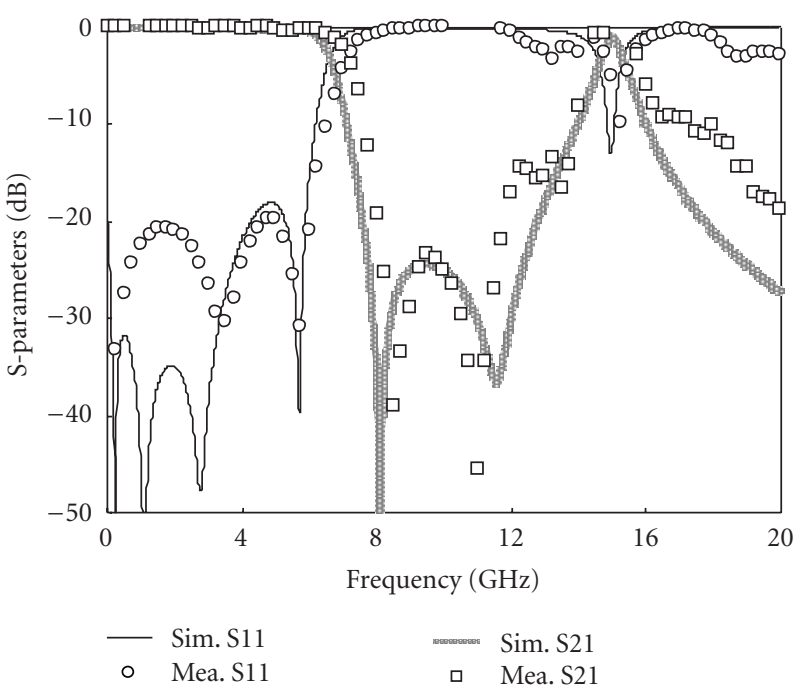

FIGURE 3: Simulated and measured frequency responses of the Sparameters of the proposed resonant cell.

is quarter wavelength long at the frequency of $2 f_{\mathrm{LO}}+f_{\mathrm{RF}}$ in order to provide a termination for the better conversion gain performance. 


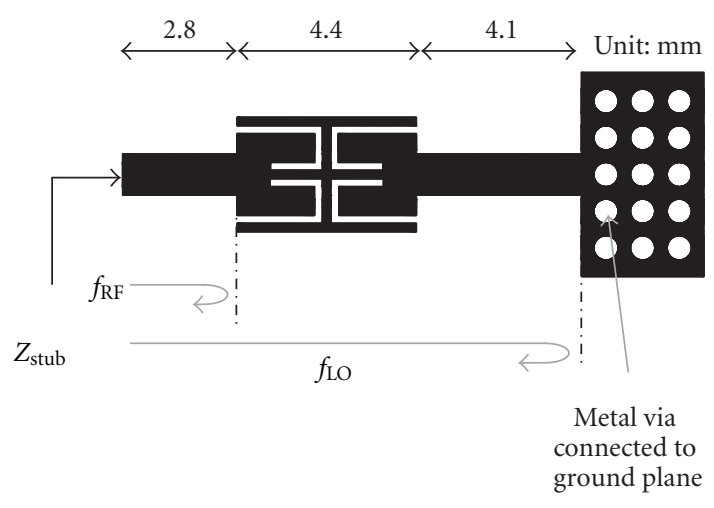

FIGURE 4: Layout of the short circuit stub incorporating resonant cell.

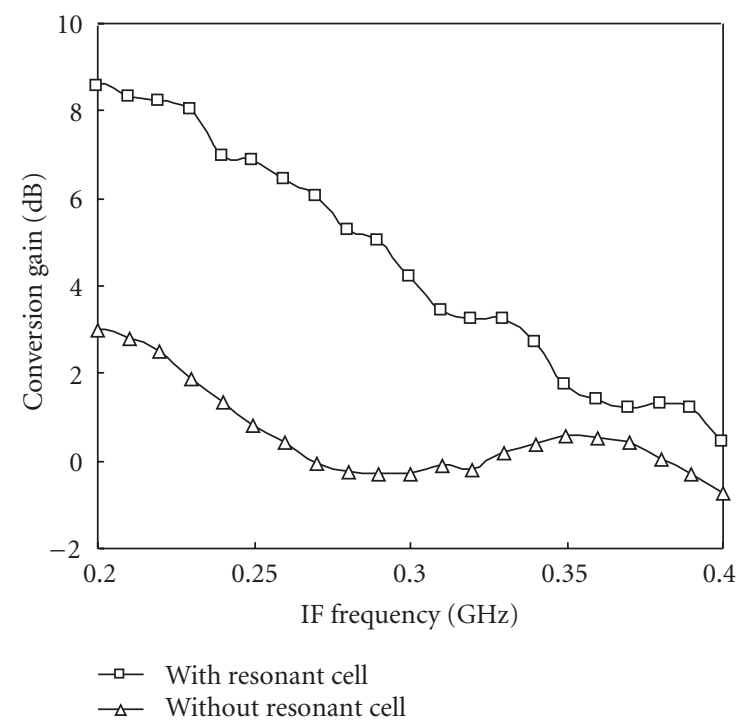

Figure 5: Measured downconversion gains of the proposed SOM and the SOM without resonant cell.

\section{Results}

The proposed SOM circuit was fabricated on a piece of RT duroid 6002 dielectric substrate with a dielectric constant of 2.94 and substrate thickness of $0.381 \mathrm{~mm}$. The active device is Infineon BFP420 NPN Silicon RF bipolar junction transistor. The commercial circuit simulator, Agilent Advanced Design System, was used to simulate and verify the SOM design. A Rohde and Schwarz FSEK30 Spectrum Analyzer was used to measure the output spectrum of the proposed SOM. The proposed SOM oscillates at the frequency of $4.0 \mathrm{GHz}$ in the measurement.

The measured frequency response of the downconversion gain against IF frequency from $0.20 \mathrm{GHz}$ to $0.40 \mathrm{GHz}$ of the proposed SOM is shown in Figure 5. A reference SOM without resonant cell was designed and measured for comparison. The maximum conversion gain of proposed SOM is $8.5 \mathrm{~dB}$ at the IF frequency of $0.2 \mathrm{GHz}$, where the reference SOM achieves $3 \mathrm{~dB}$ downconversion gain at the same IF frequency. Distinct improvement on the conversion gain within the measured frequency band is observed.

\section{Conclusion}

A harmonic SOM incorporated with resonant cell has successfully been realized. The proposed SOM exhibits distinct improvement in terms of conversion gain. The devised resonant cell not only serves as a return path for one of the major signals, but also discovers a new application in providing a series feedback of oscillating.

\section{Acknowledgments}

This work was supported in part by the Science and Technology Development Fund of Macau SAR under Grant 020/2009/A1 and by Shenzhen Science and Technology Planning Project for the Establishment of Key Laboratory in 2009 (Project CXB200903090021A).

\section{References}

[1] D. Higgins, "Performance of self-oscillating GaAs MESFET mixer at X-band," Electronics Letters, vol. 12, no. 11, pp. 605606, 1979.

[2] R. Stahlmann, C. Tsironis, F. Ponse, and H. Beneking, "Dualgate MESFET self-oscillating X-band mixers," Electronics Letters, vol. 15, no. 17, pp. 524-526, 1979.

[3] V. D. Hwang and T. Itoh, "Quasi-optical HEMT and MSEFET self-oscillating mixers," IEEE Transactions on Microwave Theory and Techniques, vol. 36, no. 12, pp. 1701-1705, 1988.

[4] M. Sironen, Y. Qian, and T. Itoh, "A subharmonic selfoscillating mixer with integrated antenna for $60-\mathrm{Ghz}$ wireless applications," IEEE Transactions on Microwave Theory and Techniques, vol. 49, no. 3, pp. 442-450, 2001.

[5] S. A. Winkler, K. Wu, and A. Stelzer, "Integrated receiver based on a high-order subharmonic self-oscillating mixer," IEEE Transactions on Microwave Theory and Techniques, vol. 55, no. 6, pp. 1398-1404, 2007.

[6] Q. Xue, K. M. Shum, and C. H. Chan, "Novel 1-D microstrip PBG cells," IEEE Microwave and Wireless Components Letters, vol. 10, no. 10, pp. 403-405, 2000. 

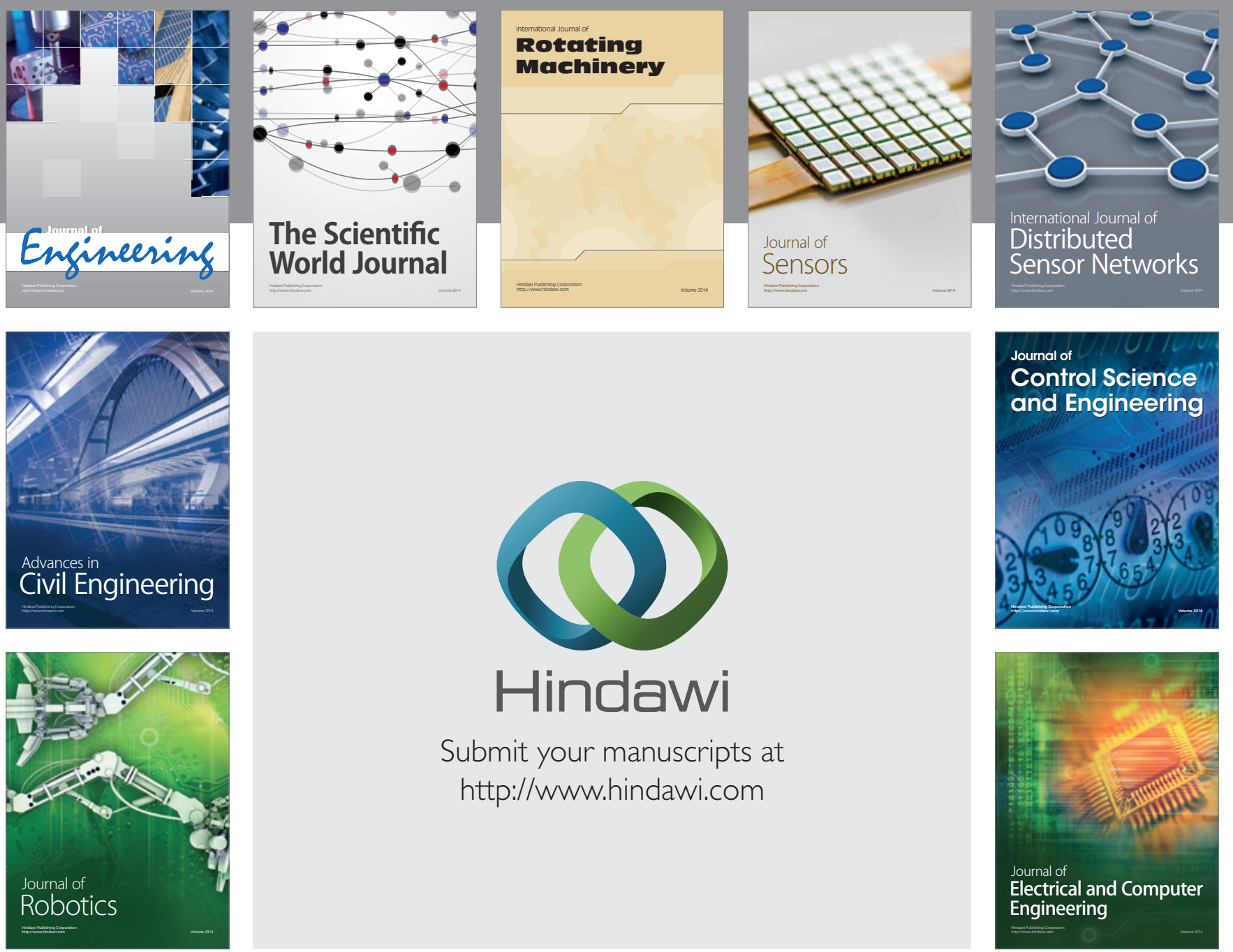

Submit your manuscripts at

http://www.hindawi.com
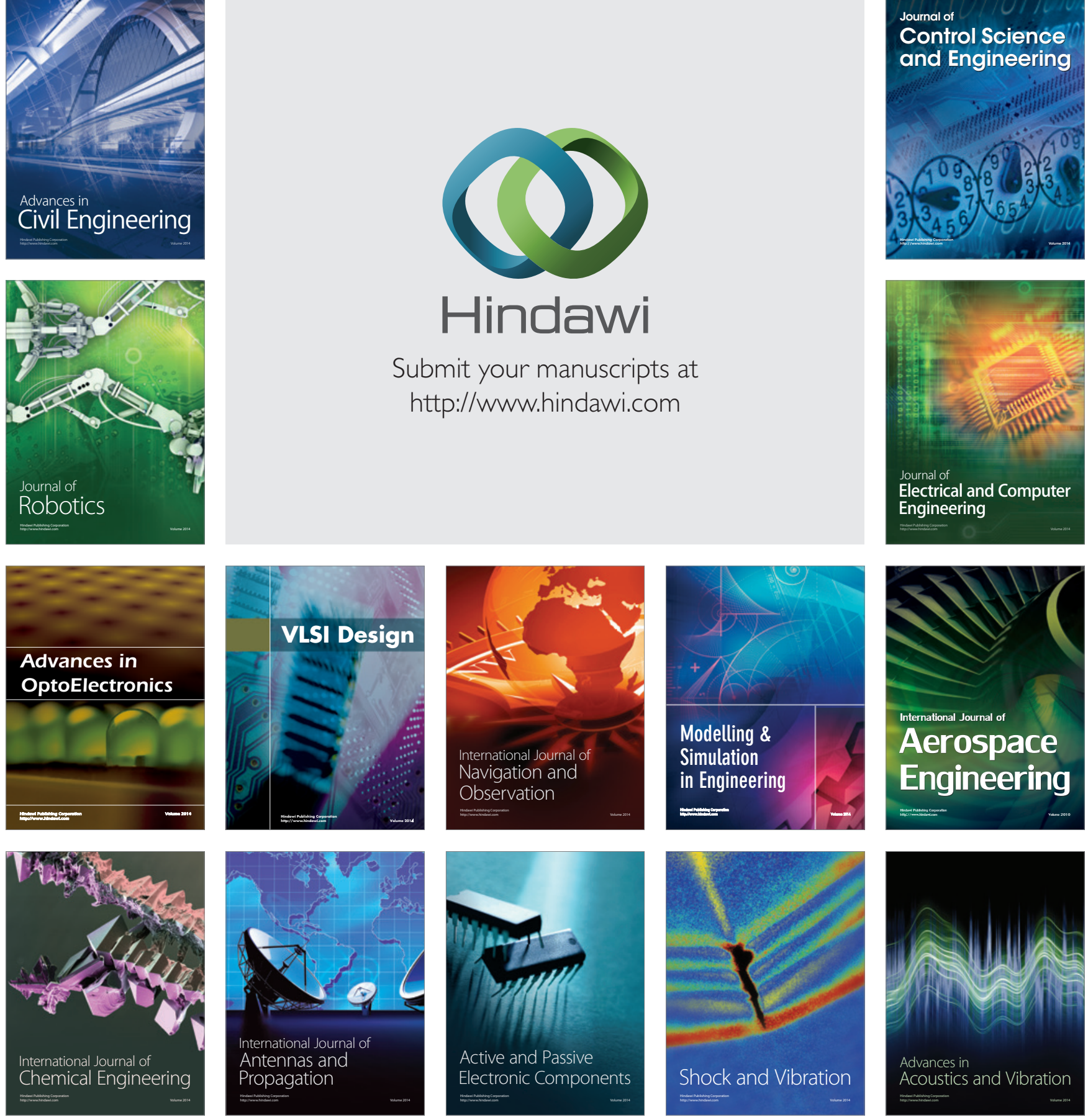